\title{
A cross-sectional analysis of the associations between leisure-time sedentary behaviors and clustered cardiometabolic risk
}

Antje Ullrich ${ }^{1,2^{*}}$, Lisa Voigt ${ }^{1,2}$, Sophie Baumann ${ }^{1,2}$, Franziska Weymar ${ }^{1,2,3}$, Ulrich John ${ }^{1,2}$, Marcus Dörr ${ }^{2,4}$ and Sabina Ulbricht ${ }^{1,2}$

\begin{abstract}
Background: The aim of this study was to conduct a comprehensive investigation of the association between different types of leisure-time sedentary behavior (watching television, using a computer, reading and socializing) and clustered cardiometabolic risk in apparently healthy adults aged 40 to 65 years.
\end{abstract}

Methods: One hundred seventy-three participants from the general population (64\% women; mean age $=54.4$ years) consented to attend a cardiovascular examination program and to complete a questionnaire on leisure-time sedentary behaviors. Waist circumference, blood pressure, glucose, triglycerides, and high-density lipoprotein cholesterol of non-fasting blood samples were assessed, and a clustered cardiometabolic risk score [CMRS] was calculated. Data were collected between February and July 2015. Associations between leisure-time sedentary behaviors and CMRS were analyzed using linear and quantile regression, adjusted for socio-demographic variables and other types of leisure-time sedentary behavior (model 1) and additionally, adjusted for leisure-time physical activity and traveling in motor vehicles (model 2).

Results: Linear regression revealed that there was a positive association between watching television and CMRS (model 1: $b=0.27$ [Cl: 0.03; 0.52]; model 2: $b=0.30$ [Cl: 0.05; 0.56]). In addition, quantile regression analysis revealed that using a computer was negatively associated with the 50th (model 1: $b=-0.43$ [Cl: -0 . 79; -0.07$]$ ) and the 75th percentiles (model 1: $b=-0.71$ [Cl: $-1.27 ;-0.14]$ ) of CMRS. Reading and socializing were not associated with CMRS.

Conclusions: Watching television was positively associated with a clustered cardiometabolic risk score, while time spent using a computer revealed inconsistent findings. Our results give reason to consider different types of behaviors in which individuals are sedentary and the associations between these behaviors and cardiometabolic risk, supporting the need for behavior-specific assessments as well as public health recommendations to maintain or enhance adults' health.

Trial registration: Clinical trial registration number: NCT02990039, Retrospectively registered (December 12, 2016).

Keywords: Sedentary behavior, Cardiometabolic risk, Linear regression, Quantile regression, Metabolic syndrome, Multiple imputation

\footnotetext{
* Correspondence: antje.ullrich@uni-greifswald.de

${ }^{1}$ University Medicine Greifswald, Institute of Social Medicine and Prevention

Walther-Rathenau-Str. 48, D-17475 Greifswald, Germany

${ }^{2}$ German Centre for Cardiovascular Research (DZHK), partner site Greifswald,

Fleischmannstr, 42-44, D-17475 Greifswald, Germany

Full list of author information is available at the end of the article
} 


\section{Background}

Sedentary behavior [SB], defined as any waking behavior characterized by an energy expenditure $\leq 1.5$ metabolic equivalents while in a sitting or reclining posture [1], is highly prevalent [2]. Prevalence has increased as utilization of media technologies in leisure time has increased. Physical activity [PA] has decreased, both at work and in leisure time [3]. There is emerging evidence that changes in patterns of PA and SB are independently related to factors of cardiometabolic health including the metabolic syndrome [2, 4]. Meta-analysis data revealed that spending high amounts of time engaging in SB increased the odds of having the metabolic syndrome by $73 \%$ compared to spending low amounts of time engaging in SB [4].

The association between leisure-time SB and factors of cardiometabolic health depends on the type of SB and the age of individuals [5]. Many studies have focused on television [TV] time or time spent using a computer and their associations with cardiovascular health $[2,6]$. Few studies have analyzed associations between other SBs such as reading or socializing in leisure time and cardiometabolic risk factors [7-12]. Time spent watching TV was found to be positively associated with obesity [10], type II diabetes [7], overweight [8, 9], or individual cardiometabolic biomarkers [10-12]. The evidence for using a computer in leisure time is inconsistent [7-12]. No associations have been found between other SBs such as reading or socializing in leisure time and cardiometabolic risk factors [7, 8].

A growing body of literature recommends using a cluster of continuous cardiometabolic risk factors instead of a binary definition of the metabolic syndrome $[13,14]$. The reasons include (i) cardiovascular risk increases progressively with an increasing number of metabolic syndrome risk factors, and (ii) using a continuous risk score increases statistical power [14].

Current evidence has suggested that a greater increase in overall sedentary time is associated with a greater increase in clustered cardiometabolic risk in adults at high risk of developing type II diabetes [15] or in a population-based sample [16]. Nevertheless, to the best of our knowledge, no study has examined a broader range of screen-based and other leisure-time SBs and their associations with clustered cardiometabolic risk. One study has analyzed associations between SBs in leisure time and two clustered cardiometabolic risk scores over a 2-year follow-up period among adults at increased cardiometabolic risk [17]. A risk score of developing type II diabetes (Atherosclerosis Risk in Communities study) and a score of developing fatal cardiovascular disease (Systematic Coronary Risk Evaluation) was used. The findings suggested no associations between time spent watching TV, using a computer, or reading and individual cardiometabolic risk factors or clustered cardiometabolic risk scores [17].

Given the ubiquitous nature of prolonged SB in leisure time, a deeper understanding is needed about whether behaviors are associated with clustered cardiometabolic risk in order to develop adequate prevention strategies. The present study aimed to examine associations between leisure-time SBs (watching TV, using the computer, and playing computer games, reading, doing household tasks, caring for others, pursuing hobbies, and socializing) and clustered cardiometabolic risk in apparently healthy adults.

\section{Methods}

\section{Selection of participants}

Participants were recruited from a sample of 1165 individuals aged 40 to 75 years who had been recruited in general medical practices, job agencies, or via a health insurance company between June 2012 and December 2013 [18], and gave consent to be contacted again (95\%). Of those, 513 persons fulfilled the following eligibility criteria: no history of cardiovascular event (myocardial infarction, stroke) or vascular intervention, age $\geq 40$ and $\leq 65$ years, self-reported body mass index $\leq 35 \mathrm{~kg} / \mathrm{m}^{2}$, and residency in a pre-defined zip-code area. A total of 401 persons were contacted and offered participation in a study that aimed to assess the feasibility of a tailored counselling letter intervention to increase PA and to reduce sedentary time.

Among those who had been offered study participation, 175 persons gave written informed consent and agreed to attend a cardiovascular examination program including the following: giving a blood sample, standardized measurement of blood pressure, waist circumference, body weight, and height at the study examination center, and completing a paper-pencil questionnaire on PA and leisure-time SB. Data were collected between February and July 2015. Two participants were excluded from analysis due to missing questionnaire or blood sample data. Our final sample comprised 173 adults (Fig. 1).

The study was approved by the clinical ethical committee of the University Medicine Greifswald (protocol number BB 002/15a).

\section{Measures}

\section{Clustered cardiometabolic risk score}

According to the definition of the American Heart Association and the National Heart, Lung, and Blood Institute [19], six cardiometabolic risk factors were assessed: waist circumference [WC], systolic blood pressure [SBP], diastolic blood pressure [DBP], glucose, plasma triglycerides, and high-density lipoprotein cholesterol [HDL-C].

WC was measured midway between the lowest rib and the iliac crest using an inelastic tape. Blood pressure was 


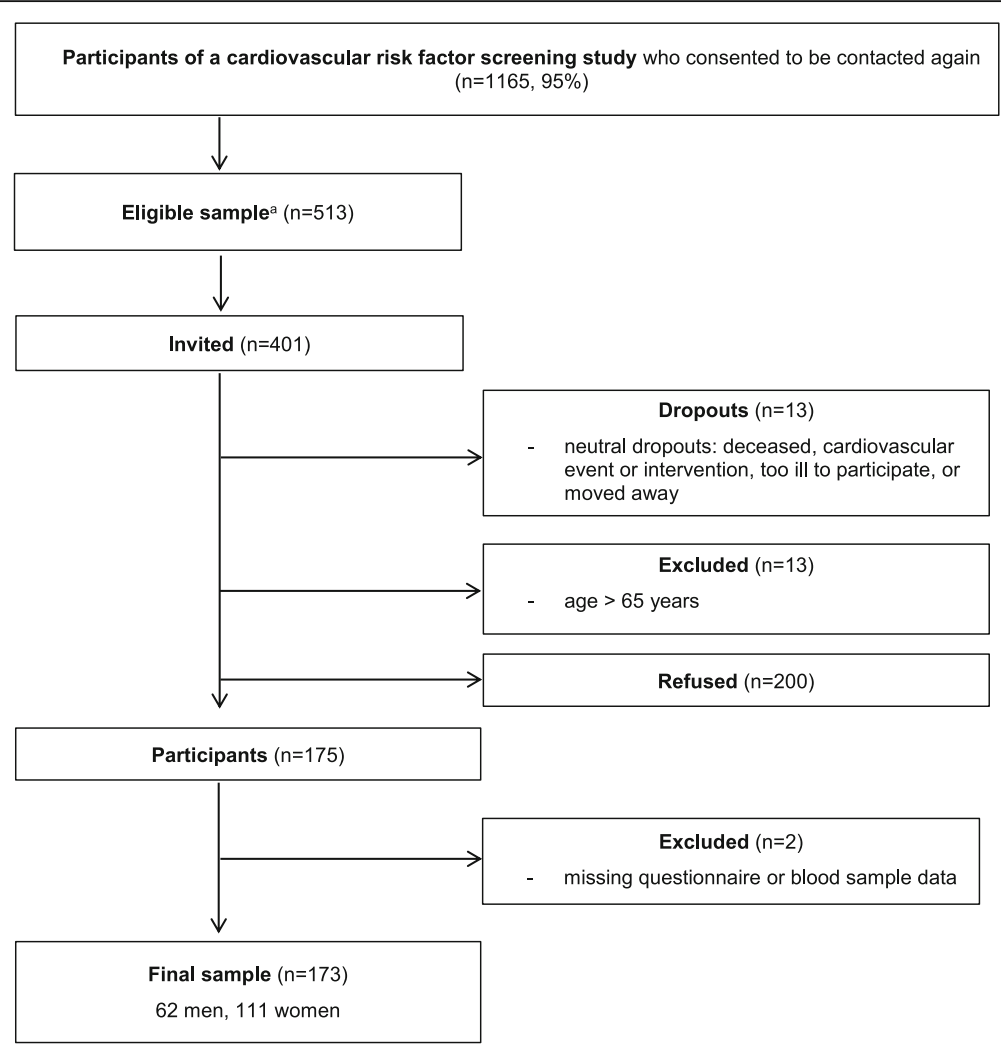

Fig. 1 Flow of participation. ${ }^{a}$ Eligibility criteria: no history of cardiovascular event (myocardial infarction, stroke), or vascular intervention, body mass index $\leq 35 \mathrm{~kg} / \mathrm{m}^{2}$, residency in a pre-defined zip-code area, and age $\geq 40$ and $\leq 65$ years

measured after a five-minute resting period three times at the right arm in a seated position using a digital blood pressure monitor (705IT, Omron Corporation, Tokyo, Japan). The first and the second reading were followed by another resting period of $3 \mathrm{~min}$ each. For analyses, the means of the second and third measurements of SBP and DBP were used. If an antihypertensive medication within the last 12 months was reported, we added $10 \mathrm{mmHg}$ to the original observed value of SBP and DBP [20]. Non-fasting blood samples were taken, and plasma triglycerides, HDL-C, and glucose were determined by standard methodology at the Institute for Clinical Chemistry and Laboratory Medicine of the University Medicine Greifswald. Values of plasma triglycerides were converted into corresponding fasting values by subtracting $3.7 \%$ of the triglyceride value per hour of non-fasting values. This calculation was made up to $8 \mathrm{~h}$ for men and up to $7 \mathrm{~h}$ for women [21].

The clustered cardiometabolic risk score [CMRS] was calculated according to Knaeps et al. [16] by the following method: glucose, triglycerides, and HDL-C were normalized $\left(\log _{10}\right)$ due to their strongly skewed distributions. Each cardiometabolic variable was standardized by using sex-specific sample means and standard deviations [SD]. We standardized the scores of WC, SBP, DBP, glucose, triglycerides, and HDL-C. Because the standardized HDL-C is inversely related to metabolic risk, it was multiplied by -1 . CMRS was created by summing the standardized values of the six cardiometabolic risk factors and dividing the sum by 6 to account for the number of variables included. A higher CMRS indicates a higher cardiometabolic risk.

\section{Sedentary behavior}

We used a modified version of the "last 7-d sedentary behavior questionnaire" ([SIT-Q-7d], Section 5: Screen time and other activities) [22] to quantify the amount of time spent in different leisure-time SBs: watching TV, using a computer, playing computer games, reading, doing household tasks, caring for others, pursuing hobbies, and socializing. Because playing computer games was rarely present in our sample $(n=25)$, we summed time spent on using a computer and on playing computer games. Average time per day in hours (h/ day) was calculated.

\section{Covariates}

Sex, age, living in a partnership (yes/ no), and employment status (employed/ unemployed) were used as covariates. To account for other health-related confounders [2], leisure-time PA in Metabolic equivalent 
of task [MET]-hours per week and time spent traveling in a motor vehicle in minutes per day were assessed using the International Physical Activity Questionnaire [IPAQ] [23] and calculated according to the IPAQ protocol. The leisure-time domain includes walking, PA on a moderate-intensity level, and PA on a vigorous-intensity level. Especially prolonged time spent sitting in cars has shown to be associated with a more-adverse cardiometabolic risk profile [24]. Therefore, the single item of the IPAQ traveling in a motor vehicle was used as covariate.

\section{Statistics}

Descriptive participant characteristics (mean $[\mathrm{M}]$ with SD and median [Med] with interquartile range [IQR]) were calculated. Leisure-time SB and PA variables were square root transformed to account for their right-skewed distributions. The constant 1 was added to the original value in order to anchor the variables at a place where square root transformation will have the optimal effect [25].

We performed multiple imputation using chained equations ( $\mathrm{m}=20$ imputed datasets) to account for missing values. The six cardiometabolic risk factors included in the outcome variable (CMRS), the predictor variables (watching $\mathrm{TV}$, using a computer, reading, and socializing), socio-demographic covariates (sex, age, living in a partnership, and employment status), PA covariates (leisure-time PA and traveling in motor vehicles), and auxiliary variables (e.g., body mass index, total cholesterol, glycated hemoglobin, low-density lipoprotein cholesterol) were considered for imputation models. To handle skewed continuous variables, we used the predictive mean matching method in the imputation procedure [26].

For analyzing associations of leisure-time SB with CMRS, we applied ordinary least-squares [OLS] regression. In regard of heteroscedasticity, we used robust standard errors estimations. Furthermore, we applied quantile regression $[Q R]$. This method is not influenced by outliers in the distribution of the outcome variable [27] and served to explore the association of different leisure-time SBs across the entire distribution of CMRS [28]. We examined associations of SB variables at the 25th, 50th, and 75th percentiles of CMRS.

For the OLS and QR analyses, adjustments were made for socio-demographics and for time spent in the other leisure-time SBs (model 1). Subsequently, we adjusted for time spent physically active in leisure time and for time spent traveling in motor vehicles (model 2). Additionally, OLS and QR analyses using complete cases of the leisure-time SB variables were calculated as a sensitivity analysis. All variables of OLS regression analyses were tested for normality and residuals were tested for homoscedasticity, linearity and independence. To diagnose multicollinearity, the variance inflation factor was calculated. Values over 5 were considered as an indication of multicollinearity [29]. $P$-values below 0.05 were considered statistically significant. Statistical analyses were performed using STATA v.14.1 software (Stata Corporation, College Station, TX) [30].

\section{Results}

\section{Characteristics of participants}

In our sample, the mean age was 54.4 years $(S D=6.2)$, $64 \%$ were women, $81 \%$ were employed, and $72 \%$ lived in a partnership (Table 1). The mean CMRS was -0.0 $(\mathrm{SD}=0.6)$ with a minimum value of -1.3 and a maximum value of 1.6 .

\section{Leisure-time sedentary behaviors}

Doing household tasks was reported by 53, caring for others by 36 , and pursuing hobbies by 87 study participants. Thus, they were excluded from the analysis and we analyzed associations of time spent watching TV, using a computer, reading, and socializing in leisure time ( $n=161, n=112, n=156$, and $n=113$, respectively, with values over zero) with CMRS.

In median, participants spent $2.5 \mathrm{~h} /$ day (IQR: $1.8-3.5$ ) watching TV, $0.4 \mathrm{~h} /$ day (IQR: 0.1-1.0) using a computer, $0.5 \mathrm{~h} /$ day (IQR: 0.4-1.0) reading, and $0.4 \mathrm{~h} /$ day (IQR: 0.0-1.0) socializing during leisure time (Table 1).

\section{Associations between leisure-time sedentary behaviors and clustered cardiometabolic risk score}

In both models, OLS regression revealed that there was a positive association between watching TV and CMRS (model 1: $b=0.27$ [CI: 0.03; 0.52]; model 2: $b=0.30$ [CI: $0.05 ; 0.56]$ ). As shown in Table 2 , QR analysis revealed that watching TV was positively associated with the 25th (model $1: b=0.35$ [CI: $0.07 ; 0.63$ ]; model $2: b=0.34$ [CI: 0.09; 0.59]), the 50th (model $1: b=0.32$ [CI: 0.02; 0.62], model 2: $b=0.37$ [CI: 0.07; 0.66]), and the 75th percentiles (model $2: b=0.32$ [CI: $0.01 ; 0.63]$ ) of CMRS. Furthermore, the 50th (model 1: $b=-0.43$ [CI: $-0.79 ;-0.07]$ ) and the 75th percentiles (model 1 : $b=-0.71$ [CI: $-1.27 ;-0.14]$ ) of CMRS revealed a negative association with using a computer. These significant associations disappeared after additionally adjusting for time spent physically active in leisure time and for time spent traveling in motor vehicles (model 2 , 50th percentile: $b=-0.28$ [CI: $-0.81 ; 0.24]$; model 2 , 75th percentile: $b=-0.55$ [CI: $-1.10 ; 0.01])$. There were no statistically significant associations between reading or socializing and CMRS. OLS and QR analyses using complete cases of the leisure-time SB variables yielded similar results (see Additional file 1: Table S1). 
Table 1 Descriptive characteristics of the study sample $(n=173)$

\begin{tabular}{|c|c|c|c|}
\hline Variables & Number & Missing data \% & Values \\
\hline \multicolumn{4}{|l|}{ Socio-demographic variables } \\
\hline Age, years (M, SD) & 173 & - & $54.4 \pm 6.2$ \\
\hline \multicolumn{4}{|l|}{ Gender (n, \%) } \\
\hline Female & 173 & - & $111(64.2)$ \\
\hline Employed (n, \%) & 170 & 2 & $138(81.2)$ \\
\hline Partnership (n, \%) & 173 & - & $124(71.7)$ \\
\hline \multicolumn{4}{|l|}{ Cardiometabolic variables } \\
\hline WC, cm (M, SD) & 172 & 1 & $91.6 \pm 12.5$ \\
\hline $\mathrm{SBP}, \mathrm{mmHg}(\mathrm{M}, \mathrm{SD})$ & 164 & 5 & $131.2 \pm 16.1$ \\
\hline $\mathrm{DBP}, \mathrm{mmHg}(\mathrm{M}, \mathrm{SD})$ & 164 & 5 & $80.7 \pm 11.0$ \\
\hline Non-fasting glucose, mmol/l (Med, IQR) & 163 & 6 & $5.3(4.9-5.8)$ \\
\hline $\mathrm{HDL}-\mathrm{C}, \mathrm{mmol} / \mathrm{l}(\mathrm{M}, \mathrm{SD})$ & 167 & 3 & $1.4 \pm 0.4$ \\
\hline Plasma triglyceride, mmol/I (Med, IQR) & 151 & 13 & $1.1(0.7-1.7)$ \\
\hline CMRS $(M, S D)$ & 170 & 2 & $-0.0 \pm 0.6$ \\
\hline \multicolumn{4}{|l|}{ Leisure-time sedentary behavior variables } \\
\hline Watching TV, h/day (Med, IQR) & 165 & 5 & $2.5(1.8-3.5)$ \\
\hline Using a computer, h/day (Med, IQR) & 142 & 18 & $0.4(0.1-1.0)$ \\
\hline Reading, h/day (Med, IQR) & 160 & 8 & $0.5(0.4-1.0)$ \\
\hline Household tasks, h/day (Med, IQR) & 154 & 11 & $0(0-0.1)$ \\
\hline Caring for others, h/day (Med, IQR) & 140 & 19 & $0(0-0.1)$ \\
\hline Hobbies, h/day (Med, IQR) & 153 & 12 & $0.1(0-0.4)$ \\
\hline Socializing, h/day (Med, IQR) & 151 & 13 & $0.4(0-1.0)$ \\
\hline \multicolumn{4}{|l|}{ Physical activity variables } \\
\hline Leisure-time physical activity, MET-h/week (Med, IQR) & 145 & 16 & $15.6(3.3-33.1)$ \\
\hline Traveling in motor vehicles, min/day (Med, IQR) & 165 & 5 & $30(10-60)$ \\
\hline
\end{tabular}

$M$ mean, $S D$ standard deviation, Med median, $I Q R$ interquartile range, WC waist circumference, $S B P$ systolic blood pressure, $D B P$ diastolic blood pressure, $H D L-C$ high-density lipoprotein cholesterol, CMRS clustered cardiometabolic risk score, TV television, MET metabolic equivalent of task

Presented are means and standard deviations for normally distributed variables, medians and interquartile ranges for non-normally distributed variables, and absolute values and percentages for categorical variables

\section{Discussion}

There were two main findings of our study. First, watching TV was positively associated with CMRS. In addition, depending on the quantiles of CMRS, QR analysis revealed a negative association between computer time and CMRS. However, this association disappeared after adjusting for PA in leisure time and time spent traveling in motor vehicles. Second, no associations were present between reading or socializing and CMRS.

Our results suggest that study participants who spend higher amounts of time watching TV are at higher cardiometabolic risk than individuals with low levels of TV time. This association remained significant after adjusting for leisure-time PA and time spent traveling in motor vehicles. Furthermore, QR analyses revealed an association between computer time and CMRS that otherwise is hidden if using the mean of CMRS in OLS regression analysis. Among study participants in the medium and in the highest cardiometabolic risk group, higher amounts of time using a computer were associated with a more favorable cardiometabolic profile. However, this association disappeared after adjusting for leisure-time PA and time spent traveling in motor vehicles.

Our findings on associations between TV time and CMRS are in line with current evidence on associations between TV time and individual cardiometabolic risk factors [12, 10, 2]. Additionally, watching TV has been shown to be associated with lower energy expenditure [31], an increased intake of food with high energy density and overall unhealthy dietary habits [32, 33] compared with other sedentary activities such as using a computer. A combination of these factors may explain our findings $[12,10]$. According to the QR result, time spent sedentary while using a computer may be differentially associated with CMRS. Whereas a populationbased study suggested no association between using a computer in leisure time and individual risk factors of 
Table 2 Results of linear and quantile regression of multiply imputed data ( $n=173$ )

\begin{tabular}{|c|c|c|c|c|c|c|c|c|}
\hline & OLS & & QR25 & & QR50 & & QR75 & \\
\hline $\mathrm{CMRS}^{\mathrm{a}}$ & $b[95 \% \mathrm{Cl}]$ & $p^{\mathrm{b}}$ & $b[95 \% \mathrm{Cl}]$ & $p^{\mathrm{b}}$ & $b[95 \% \mathrm{Cl}]$ & $p^{\mathrm{b}}$ & $b[95 \% \mathrm{Cl}]$ & $p^{\mathrm{b}}$ \\
\hline \multicolumn{9}{|l|}{ Watching TV } \\
\hline Model $1^{c}$ & $0.27^{*}[0.03 ; 0.52]$ & 0.029 & $0.35^{*}[0.07 ; 0.63]$ & 0.015 & $0.32^{*}[0.02 ; 0.62]$ & 0.039 & $0.26[-0.09 ; 0.62]$ & 0.143 \\
\hline Model $2^{d}$ & $0.30^{*}[0.05 ; 0.56]$ & 0.021 & $0.34^{* *}[0.09 ; 0.59]$ & 0.008 & $0.37^{*}[0.07 ; 0.66]$ & 0.015 & $0.32^{*}[0.01 ; 0.63]$ & 0.041 \\
\hline \multicolumn{9}{|c|}{ Using a computer } \\
\hline Model $1^{c}$ & $-0.35[-0.70 ; 0.00]$ & 0.051 & $-0.15[-0.67 ; 0.18]$ & 0.251 & $-0.43^{*}[-0.79 ;-0.07]$ & 0.019 & $-0.71^{*}[-1.27 ;-0.14]$ & 0.015 \\
\hline Model $2^{d}$ & $-0.26[-0.65 ; 0.13]$ & 0.188 & $-0.26[-0.66 ; 0.13]$ & 0.191 & $-0.28[-0.81 ; 0.24]$ & 0.277 & $-0.55[-1.10 ; 0.01]$ & 0.052 \\
\hline \multicolumn{9}{|l|}{ Reading } \\
\hline Model $1^{c}$ & $-0.07[-0.57 ; 0.42]$ & 0.766 & $-0.18[-0.71 ; 0.35]$ & 0.502 & $-0.21[-0.95 ; 0.53]$ & 0.573 & $0.06[-0.72 ; 0.83]$ & 0.888 \\
\hline Model $2^{d}$ & $-0.17[-0.68 ; 0.34]$ & 0.515 & $-0.36[-0.84 ; 0.13]$ & 0.145 & $-0.33[-1.17 ; 0.50]$ & 0.429 & $-0.04[-0.77 ; 0.70]$ & 0.922 \\
\hline \multicolumn{9}{|l|}{ Socializing } \\
\hline Model $1^{c}$ & $-0.08[-0.38 ; 0.21]$ & 0.578 & $0.07[-0.33 ; 0.48]$ & 0.718 & $-0.09[-0.44 ; 0.26]$ & 0.616 & $-0.26[-0.72 ; 0.19]$ & 0.255 \\
\hline Model $2^{d}$ & $-0.06[-0.37 ; 0.24]$ & 0.687 & $0.08[-0.23 ; 0.38]$ & 0.619 & $-0.07[-0.46 ; 0.31]$ & 0.708 & $-0.20[-0.64 ; 0.24]$ & 0.374 \\
\hline
\end{tabular}

$O L S$ ordinary least squares regression, $Q R$ quantile regression, $b$ unstandardized regression coefficient, $C I$ confidence interval, $T V$ television

a Presented are multiply imputed data using chained equations ( $m=20$ imputed datasets) to account for missing values

${ }^{b}$ Based on robust standard errors, ${ }^{* *} p<0.01,{ }^{*} p<0.05$

' Model 1: Adjusted for socio-demographic (sex, age, partnership, and employment) and other leisure-time sedentary behavior variables

d Model 2: Adjusted for socio-demographic (sex, age, partnership, and employment), leisure-time physical activity, traveling in motor vehicles, and other leisure-time sedentary behavior variables

cardiometabolic health [11], Heinonen et al. [10] reported a positive association between computer time and WC as well as body mass index among women but not among men in a middle-aged sample. In contrast to these studies, our results of QR revealed a negative association between using a computer and CMRS among individuals in the medium and in the highest cardiometabolic risk group. After adjusting for two PA variables, the statistically significant association between using a computer and CMRS disappeared whereas the negative direction of the association remained. Thus, different behaviors in which individuals spend their time in a sitting or reclining posture may not influence the magnitude and direction of the association with clustered cardiometabolic risk in the same manner.

Our second finding adds to the literature that there seems to be no association between reading and socializing and clustered cardiometabolic risk. This finding is in line with previous studies that examined associations between time spent sedentary while reading or socializing and individual cardiometabolic health factors [7-12, 17]. The less time spent reading or socializing in leisure time might be an explanation for the findings in our sample. In addition, accuracy to recall across contexts may vary [2] in the sense that it may be easier for people to remember how long they have watched TV than how much time they have spent reading or in company with others [34].

Furthermore, we found that leisure-time SBs differed in their frequency of occurrence. Time spent sitting while doing household tasks, caring for others, or pursuing hobbies were less prevalent in our sample. Evidence suggests that different types of SB often cooccur compared to activities with higher energy expenditure (over 1.5 metabolic equivalents) [22], e.g. using the computer or doing household tasks while watching TV. Thus, it is important to consider not only the frequency of occurrence but also that of co-occurrence of leisuretime SB and how different types of leisure-time SB are linked to one another. Future studies should examine those patterns of leisure-time SB in detail and might include separate analyses for weekdays and weekends, because leisure-time SB patterns have been shown to vary between weekends and weekdays [35].

Some limitations of our study have to be discussed. First, subjects were assessed within a study aiming to test the feasibility of a tailored letter intervention regarding PA and leisure-time SB. The proportion of people who declined participation (53\%) was high and a selection bias is likely. Thus, our findings may not be generalizable to the population as a whole. Second, there may be confounding variables such as diet, drinking habits, different activity patterns with certain energy expenditure during leisure-time SBs, sleep duration, or cardiorespiratory fitness that were not considered in our study. Third, we collected blood samples in the nonfasted state. Because levels of glucose or HDL-C are influenced by external factors like caloric intake or muscle activity [36], this may have implications for the clustered cardiometabolic risk. Although using fasting blood samples is recommended, there is evidence that using nonfasting blood samples is appropriate for decision making 
in the context of primary preventions regarding cardiovascular or cardiometabolic diseases [36, 37]. Fourth, we assessed leisure-time SB by self-report. Self-report assessments are sensitive to recall bias and social desirability [2]. In comparison to accelerometer measures of SB, self-report appears to capture different aspects of behaviors [38] because it provides information on the context. Keeping in mind that leisure-time SB is complex and includes multiple domains, dimensions, and correlates, there are still many methodological challenges of measuring leisure-time SB [39]. Finally, the design of our study does not allow for causal inference. To address this issue, more longitudinal studies are needed to understand the directionality of potential associations between leisure-time SBs and cardiometabolic health.

\section{Conclusions}

Watching TV was positively associated with a clustered cardiometabolic risk score, while results of time spent using a computer revealed inconsistent findings. No associations were present between reading or socializing and clustered cardiometabolic risk.

Our findings suggest that different leisure-time SBs and their differential associations with cardiometabolic risk should be considered. This approach would address the needs (i) for behavior specific assessments, (ii) to develop relevant public health recommendations and guidelines to maintain or enhance adults' health, and (iii) to encourage environmental and policy initiatives and interventions [2].

\section{Additional file}

Additional file 1: Table S1. Results of linear and quantile regression of complete cases. (DOCX $20 \mathrm{~kb}$ )

\section{Abbreviations \\ Cl: Confidence interval; CMRS: Clustered cardiometabolic risk score: DBP: Diastolic blood pressure; HDL-C: High-density lipoprotein cholesterol; IPAQ: International physical activity questionnaire; IQR: Interquartile range; M: Mean; Med: Median; MET: Metabolic equivalent of task; OLS: Ordinary least-squares regression; PA: Physical activity; QR: Quantile regression; SB: Sedentary behavior; SBP: Systolic blood pressure; SD: Standard deviation; SIT-Q-7d: Last 7-d sedentary behavior questionnaire; TV: Television; WC: Waist circumference}

\section{Acknowledgements}

The authors wish to thank funders, supporters, and participants of the study. Further, we thank Liane Müller and Ramona Mühlenbächer for their assistance in screening, recruitment, and with data collection.

\section{Funding}

This study was funded by the Federal Ministry of Education and Research as part of the German Centre for Cardiovascular Research, DZHK (grant no. 81/Z540100152).

\section{Availability of data and materials}

The datasets analyzed during the current study are available from the corresponding author on reasonable request.

\section{Authors' contributions}

FW, UJ, MD, and SU contributed to the conception or design of the study. All authors contributed to the acquisition, analysis, or interpretation of data for the work. AU drafted the manuscript. LV, SB, FW, UJ, MD, and SU critically revised the manuscript. All authors gave final approval and agreed to be accountable for all aspects of work, thus ensuring integrity and accuracy.

\section{Ethics approval and consent to participate}

All procedures performed in studies involving human participants were in accordance with the ethical standards of the institutional and/or national research committee and with the 1964 Helsinki Declaration and its later amendments or comparable ethical standards.

This study was approved by the clinical ethical committee of the University Medicine Greifswald, Germany (protocol number BB 002/15a) and retrospectively registered to the ClinicalTrials.gov Protocol Registration and Results System (Clinical trial registration number: NCT02990039). Informed written consent was obtained from all individual participants included in the study.

\section{Consent for publication}

Not applicable.

\section{Competing interests}

The authors declare that they have no competing interests.

\section{Publisher's Note}

Springer Nature remains neutral with regard to jurisdictional claims in published maps and institutional affiliations.

\section{Author details}

${ }^{1}$ University Medicine Greifswald, Institute of Social Medicine and Prevention, Walther-Rathenau-Str. 48, D-17475 Greifswald, Germany. ${ }^{2}$ German Centre for Cardiovascular Research (DZHK), partner site Greifswald, Fleischmannstr, 42-44, D-17475 Greifswald, Germany. ${ }^{3}$ University Medicine Greifswald, Institute for Community Medicine, Section Epidemiology of Health Care and Community Health, Ellernholzstr, 1-2, D-17487 Greifswald, Germany. ${ }^{4}$ University Medicine Greifswald, Department of Internal Medicine B, Ferdinand-Sauerbruch-Str, D-17475 Greifswald, Germany.

Received: 11 September 2017 Accepted: 23 February 2018

Published online: 06 March 2018

\section{References}

1. Sedentary Behaviour Research N. Letter to the editor: standardized use of the terms "sedentary" and "sedentary behaviours". Appl Physiol Nutr Metab. 2012;37(3):540-2. https://doi.org/10.1139/h2012-024.

2. Young DR, Hivert M-F, Alhassan S, Camhi SM, Ferguson JF, Katzmarzyk PT, et al. Sedentary behavior and cardiovascular morbidity and mortality: a science advisory from the American Heart Association. Circulation. 2016; 134(13):e262-e79. https://doi.org/10.1161/cir.0000000000000440.

3. Ng SW, Popkin B. Time use and physical activity: a shift away from movement across the globe. Obes Rev. 2012;13(8):659-80. https://doi.org/10.1111/j.1467-789X.2011.00982.x.

4. Edwardson CL, Gorely T, Davies MJ, Gray LJ, Khunti K, Wilmot EG, et al. Association of Sedentary Behaviour with metabolic syndrome: a meta-analysis. PLoS One. 2012;7(4):e34916. https://doi.org/10.1371/journal.pone.0034916.

5. de Rezende LFM, Rodrigues Lopes M, Rey-López JP, Matsudo VKR, OdC L. Sedentary behavior and health outcomes: an overview of systematic reviews. PLoS One. 2014;9(8):e105620. https://doi.org/10.1371/journal.pone.0105620.

6. Mansoubi M, Pearson N, Biddle SJH, Clemes S. The relationship between sedentary behaviour and physical activity in adults: a systematic review. Prev Med. 2014;69:28-35. https://doi.org/10.1016/j.ypmed.2014.08.028.

7. Hsueh M-C, Liao Y, Chang S-H. Associations of Total and domain-specific sedentary time with type 2 diabetes in Taiwanese older adults. J. Epidemiol. 2016;26(7):348-54. https://doi.org/10.2188/jea.JE20150095.

8. Hsueh M-C, Liao Y, Chang S-H. Are Total and domain-specific sedentary time associated with overweight in older Taiwanese adults? Int J Environ Res Public Health. 2015;12(10):12697-705. https://doi.org/10.3390/ijerph121012697.

9. Kikuchi H, Inoue S, Sugiyama T, Owen N, Oka K, Nakaya T, et al. Distinct associations of different sedentary behaviors with health-related attributes among older adults. Prev Med. 2014;67:335-9. https://doi.org/10.1016/j. ypmed.2014.08.011. 
10. Heinonen I, Helajärvi H, Pahkala K, Heinonen OJ, Hirvensalo M, Pälve K, et al. Sedentary behaviours and obesity in adults: the cardiovascular risk in young Finns study. BMJ Open. 2013;3(6):e002901. https://doi.org/10.1136/bmjopen2013-002901.

11. Nang EEK, Salim A, Wu Y, Tai ES, Lee J, Van Dam RM. Television screen time, but not computer use and reading time, is associated with cardio-metabolic biomarkers in a multiethnic Asian population: a cross-sectional study. Int. J. Behav. Nutr.Phys. Act. 2013:10:70. https://doi.org/10.1186/1479-5868-10-70.

12. Bertrais S, Beyeme-Ondoua JP, Czernichow S, Galan P, Hercberg S, Oppert JM. Sedentary behaviors, physical activity, and metabolic syndrome in middle-aged French subjects. Obes Res. 2005;13(5):936-44. https://doi.org/10.1038/oby.2005.108.

13. Beckstead JW, Beckie TM. How much information can metabolic syndrome provide? An application of information theory. Med Decis Mak. 2011;31(1): 79-92. https://doi.org/10.1177/0272989x10373401.

14. Kahn R, Buse J, Ferrannini E, Stern M. The metabolic syndrome: time for a critical appraisal: joint statement from the American Diabetes Association and the European Association for the Study of diabetes. Diabetes Care. 2005;28(9):2289-304.

15. Wijndaele K, Orrow G, Ekelund U, Sharp SJ, Brage S, Griffin SJ, et al. Increasing objectively measured sedentary time increases clustered cardiometabolic risk: a 6 year analysis of the ProActive study. Diabetologia. 2014;57(2):305-12. https://doi.org/10.1007/s00125-013-3102-y.

16. Knaeps S, Bourgois JG, Charlier R, Mertens E, Lefevre J, Wijndaele K. Ten-year change in sedentary behaviour, moderate-to-vigorous physical activity, cardiorespiratory fitness and cardiometabolic risk: independent associations and mediation analysis. Br J Sports Med. 2016; https://doi.org/10.1136/ bjsports-2016-096083.

17. Altenburg TM, Lakerveld J, Bot SD, Nijpels G, Chinapaw MJ. The prospective relationship between sedentary time and cardiometabolic health in adults at increased cardiometabolic risk - the Hoorn prevention study. Int J Behav Nutr Phys Act. 2014;11:90. https://doi.org/10.1186/s12966-014-0090-3.

18. Guertler D, Meyer C, Dorr M, Braatz J, Weymar F, John U, et al. Reach of individuals at risk for cardiovascular disease by proactive recruitment strategies in general practices, job centers, and health insurance. Int J. Behav. Med. 2017;24(1):153-60. https://doi.org/10.1007/s12529-016-9584-5.

19. Grundy SM. Metabolic syndrome scientific statement by the American Heart Association and the National Heart, Lung, and Blood Institute. Arterioscler Thromb Vasc Biol. 2005;25(11):2243-4. https://doi.org/10.1161/01.ATV. 0000189155.75833.c7.

20. Tobin MD, Sheehan NA, Scurrah KJ, Burton PR. Adjusting for treatment effects in studies of quantitative traits: antihypertensive therapy and systolic blood pressure. Stat Med. 2005;24(19):2911-35. https://doi.org/10.1002/sim.2165.

21. Sundvall J, Leiviska J, Laatikainen T, Peltonen M, Salomaa V, Vanhala M, et al. The use of fasting vs. non-fasting triglyceride concentration for estimating the prevalence of high LDL-cholesterol and metabolic syndrome in population surveys. BMC Med Res Methodol. 2011;11:63. https://doi.org/10.1186/1471-2288-11-63.

22. Wijndaele K, De Bourdeaudhuij I, Godino JG, Lynch BM, Griffin SJ, Westgate $\mathrm{K}$, et al. Reliability and validity of a domain-specific last 7-d sedentary time questionnaire. Med Sci Sports Exerc. 2014;46(6):1248-60.

23. Craig CL, Marshall AL, Sjostrom M, Bauman AE, Booth ML, Ainsworth BE, et al. International physical activity questionnaire: 12-country reliability and validity. Med Sci Sports Exerc. 2003;35(8):1381-95. https://doi.org/10.1249/01.MSS.0000078924.61453.FB.

24. Sugiyama T, Wijndaele K, Koohsari MJ, Tanamas SK, Dunstan DW, Owen N. Adverse associations of car time with markers of cardio-metabolic risk. Prev Med. 2016;83:26-30. https://doi.org/10.1016/j.ypmed.2015.11.029.

25. Osborne J. Notes on the use of data transformations. Pract. Assess., Res. Eval. 2002;8(6):1-7.

26. White IR, Royston P, Wood AM. Multiple imputation using chained equations: issues and guidance for practice. Stat Med. 2011;30(4):377-99. https://doi.org/10.1002/sim.4067.

27. Bottai M, Frongillo EA, Sui X, O'Neill JR, McKeown RE, Burns TL, et al. Use of quantile regression to investigate the longitudinal association between physical activity and body mass index. Obesity (Silver Spring, Md). 2014; 22(5):E149-56. https://doi.org/10.1002/oby.20618.

28. Koenker R. Quantile regression. Econometric society monographs. Cambridge: Cambridge University Press; 2005.

29. Menard S. Applied logistic regression analysis. 2nd ed. Thousand Oaks: SAGE Publications; 02002.
30. StataCorp. Stata statistical software: release 14.1. TX: StataCorp LP, College Station; 2015.

31. Hamilton MT, Hamilton DG, Zderic TW. Role of low energy expenditure and sitting in obesity, metabolic syndrome, type 2 diabetes, and cardiovascular disease. Diabetes. 2007;56(11):2655-67. https://doi.org/10.2337/db07-0882.

32. Compernolle S, De Cocker K, Teixeira PJ, Oppert J-M, Roda C, Mackenbach $J \mathrm{D}$, et al. The associations between domain-specific sedentary behaviours and dietary habits in European adults: a cross-sectional analysis of the SPOTLIGHT survey. BMC Public Health. 2016;16:1057. https://doi.org/10.1186/ s12889-016-3708-3.

33. Pearson N, Biddle SJ. Sedentary behavior and dietary intake in children, adolescents, and adults. A systematic review. Am J Prev Med. 2011;41(2): 178-88. https://doi.org/10.1016/j.amepre.2011.05.002.

34. Clark BK, Sugiyama T, Healy GN, Salmon J, Dunstan DW, Owen N. Validity and reliability of measures of television viewing time and other nonoccupational sedentary behaviour of adults: a review. Obes Rev. 2009;10(1): 7-16. https://doi.org/10.1111/j.1467-789X.2008.00508.x.

35. Prince SA, Reed JL, McFetridge C, Tremblay MS, Reid RD. Correlates of sedentary behaviour in adults: a systematic review. Obes Rev. 2017;18(8): 915-35. https://doi.org/10.1111/obr.12529.

36. Moebus S, Göres L, Lösch C, Jöckel K-H. Impact of time since last caloric intake on blood glucose levels. Eur J Epidemiol. 2011;26(9):719-28. https://doi.org/10.1007/s10654-011-9608-z.

37. Craig SR, Amin RV, Russell DW, Paradise NF. Blood cholesterol screening influence of fasting state on cholesterol results and management decisions. J Gen Intern Med. 2000;15(6):395-9.

38. Honda T, Chen S, Kishimoto H, Narazaki K, Kumagai S. Identifying associations between sedentary time and cardio-metabolic risk factors in working adults using objective and subjective measures: a cross-sectional analysis. BMC Public Health. 2014;14(1):1307. https://doi.org/10.1186/1471-2458-14-1307.

39. Kelly P, Fitzsimons C, Baker G. Should we reframe how we think about physical activity and sedentary behaviour measurement? Validity and reliability reconsidered. Int J Behav Nutr Phys Act. 2016;13:32. https://doi.org/10.1186/s12966-016-0351-4.

\section{Submit your next manuscript to BioMed Central and we will help you at every step:}

- We accept pre-submission inquiries

- Our selector tool helps you to find the most relevant journal

- We provide round the clock customer support

- Convenient online submission

- Thorough peer review

- Inclusion in PubMed and all major indexing services

- Maximum visibility for your research

Submit your manuscript at www.biomedcentral.com/submit
Biomed Central 